\section{Acute monocytic leukemia diagnosed by flow cytometry includes acute myeloid leukemias with weakly or faintly positive non-specific esterase staining}

\author{
Yuriko Zushi, ${ }^{1}$ Miho Sasaki, ${ }^{1}$ \\ Ayano Mori, ${ }^{1}$ Toshiharu Saitoh, ${ }^{1}$ \\ Takae Goka, ${ }^{2}$ Yumi Aoyama, ${ }^{3}$ \\ Yuta Goto, ${ }^{3}$ Hiroko Tsunemine, ${ }^{3}$ \\ Taiichi Kodaka, ${ }^{3}$ Takayuki Takahashi ${ }^{3}$ \\ Departments of ${ }^{1}$ Cell Therapy, \\ ${ }^{2}$ Laboratory Medicine, and \\ ${ }^{3}$ Hematology, Shinko Hospital, Kobe, \\ Japan
}

\begin{abstract}
A diagnosis of acute monocytic leukemia (AML-M5) based on $\alpha$-naphthyl butyrate esterase $(\alpha-\mathrm{NB})$ staining has some problems, because AML-M5 leukemic cells often show weak or faint positivity on $\alpha$-NB staining. In these situations, some cases of AML-M5 tend to be misdiagnosed as AML-M0. Therefore, we evaluated the significance of weak or faint $\alpha$-NB staining in AML-M5 diagnosed by flow cytometry (FCM). Nineteen AML cases in which leukemic cells were negative for naphthol AS-D chloroacetate esterase staining were studied. For FCM, we defined leukemic cells as having a monocytic nature when more than $10 \%$ of the leukemic cells were positive for at least one of the following antigens: CD4, CD11c, CD14, and CD64. The monocytic nature determined by FCM was consistent with positive or weak positivity on $\alpha$-NB staining. Five of 6 cases in which leukemic cells exhibited faint positivity for $\alpha$-NB staining could be diagnosed as AML-M5 by FCM, while negative $\alpha$-NB staining was consistent with a diagnosis of AML-M0. These results suggest that AML-M5 should be taken into consideration even when leukemic cells are faintly positive for $\alpha-\mathrm{NB}$ staining.
\end{abstract}

\section{Introduction}

It is sometimes difficult to make a differential diagnosis of acute monocytic leukemia (AML-M5 by FAB Classification) from AML-M0 (FAB Classification), which shows poor differentiation in morphological and cytochemical examinations, because leukemic cells from AML-M5 are often negative for myeloperoxidase (MPO) staining. These cells also frequently show weak or faint positivity on $\alpha$-naphthyl butyrate esterase $(\alpha-\mathrm{NB})$ staining, which is a specific tool to determine if leukemic cells have a monocytic nature. ${ }^{1-3}$ Furthermore, AMLM5 cells are sometimes negative for cytoplasmic MPO (cyMPO) on flow cytometric (FCM) analysis. ${ }^{4-6}$ Weak or faint positivity often causes a diagnostic problem in which AML M5 is misdiagnosed as AML-M0 because of the controversial interpretation regarding the weak or faint positivity of $\alpha$ NB staining. Therefore, we conducted a study to evaluate the significance of weak or faint $\alpha$-NB staining in patients diagnosed with AML-M5 by FCM.

Surface antigens that indicate leukemic cells to be of a monocytic nature include CD4, CD11c, CD14, and CD64. Immature and mature monocytes express CD64, ,-15 $^{-15}$

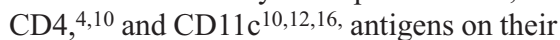
surface membranes, whereas CD145-11, 13-16 is mostly expressed on mature monocytes. However, all of these antigens are not simultaneously expressed on leukemic cells in a single AML-M5 case; sole or dual expression of these antigens appears to be common. Therefore, in this study, we considered that single as well as multiple expressions of these antigens is sufficient to make a diagnosis of AML-M5 by FCM. Thus, in this study, we examined the relationship between the expression of these antigens and $\alpha$-NB staining.

\section{Materials and Methods}

\section{Patients}

Between January 2012 and November 2016, 19 patients in whom AML-M5 was suspected were studied. This retrospective study was approved by the institutional review board. In all patients, except Patients 14 and 18, bone marrow aspirates, which were obtained for diagnostic purposes, were analyzed by FCM and cytochemistry. In Patients 14 and 18, peripheral blood was examined upon presentation. These analyses were performed as part of the diagnostic process, not for the clinical trial, to determine the AML subtype.

\section{Cytochemistry}

Marrow or peripheral blood samples were evaluated with a kit for double esterase staining (Muto Pure Chemicals, Tokyo, Japan). All patients with AML were included in this study regardless of positive or negative MPO staining of leukemic cells. On the other hand, patients in whom
Correspondence: Takayuki Takahashi, Department of Hematology, Shinko Hospital, 1-4-47, Wakihama-cho, Chuo-ku, Kobe 6510072, Japan.

Tel.: +81.78.261.6711 - Fax: +81.78.261.6726

E-mail: takahashi.takayuki@shinkohp.or.jp

Key words: AML-M5, AML-M0, flow cytometry, $\alpha$-naphthyl butyrate esterase staining, surface monocytic antigen.

Contributions: The authors contributed equally.

Conflict of interest: The authors declare no potential conflict of interest.

Received for publication: 5 October 2017

Revision received: 10 February 2018.

Accepted for publication: 28 February 2018.

This work is licensed under a Creative Commons Attribution-NonCommercial 4.0 International License (CC BY-NC 4.0).

(C) Copyright Y. Zushi et al., 2018

Licensee PAGEPress, Italy

Hematology Reports 2018; 10:7435

doi:10.4081/hr.2018.7435

leukemic cells were positive for naphthol AS-D chloroacetate esterase staining, which is specific for the neutrophilic cell lineage, ${ }^{1}$ were excluded from this study. Also, patients with AML-M6 or AML-M7 were excluded. To the best of our knowledge, no study has defined the $\alpha$-NB activity required to make a diagnosis of monocytic leukemia. We did, however, find the diagnostic criteria for $\alpha$-NB staining on the website for Beckman Coulter, Inc. (Tokyo, Japan). We show the criteria, which were originally written in Japanese, as Table 1. According to the criteria, leukemic cells with more than 6 dots in their cytoplasm are considered to show positive staining $(+)$, but when the dot number is less than 5 , it is considered weak or faint staining $( \pm$ ) (Table 1). However, from our preliminary observations, we thought that the weak or faint criteria ( 1 to 5 dots) were ambiguous, leading to diagnostic confusion as to whether it was AML-M5 or AML-M0. Therefore, in the present study, we defined the positivity of $\alpha$-NB staining as follows (Table 1): positive staining $(++)$ : more than $3 \%$ of leukemic cells contain more than 6 dots in the cytoplasm (Figure 1A); weakly positive $(+)$ : leukemic cells with 3 to 5 dots (Figure 1B); faintly positive $( \pm)$ : those with 1 or 2 dots (Figure 1C); negative (-): those without cytoplasmic dots. We excluded small cells with round nuclei in the evaluation of the $\alpha$ NB staining because normal T lymphocytes show weak positivity on $\alpha$-NB staining. ${ }^{2}$ 


\section{Flow cytometry}

Flow cytometry was performed using FACS Cant II (Nippon Becton Dickinson Company, Ltd. (Tokyo, Japan) according to the manufacturer's instructions. All marrow and blood samples were analyzed by CD45positive mononuclear cell (MNC) gating as the first step. In this study, we diagnosed the leukemic cells as having a monocytic nature when the cells expressed either CD4, CD11c, CD14, or CD64 in more than $10 \%$ of the analyzed cells in addition to an AML pattern (positive for CD13, CD33, and CD34). We also defined acute leukemias as AML-M0 on flow cytometric analysis when leukemic cells showed the AML but not lymphoid pattern with or without cyMPO and without antigens related to monocytic, erythroid, or megakaryocytic lineages. Monoclonal antibodies employed in this study were as follows: CD45 (PerCP), CD4 (FITC), CD11b (PE), CD11c (FITC), CD13 (PE), CD14 (FITC), CD19 (APC), CD33 (PE), CD34 (APC), CD56 (APC), CD64 (FITC), and MPO (FITC). The MPO antibody was purchased from Beckman Coulter Co., Inc. (Tokyo, Japan) and the others were from Nippon Becton Dickinson Company, Ltd. The time of incubation with each antibody was $15 \mathrm{~min}$ at room temperature, then red blood cells were lysed in phosphate buffer solution (PBS) containing 0.5\% paraformaldehyde and $0.5 \%$ saponin for 5

Table 1. $\alpha$-NB esterase activity criteria for the diagnosis of monocytic leukemia.

\begin{tabular}{|c|c|}
\hline Original criteria & \\
\hline Positive $(+)$ & More than 6 dots of $\alpha$-NB esterase \\
\hline Weak or Faint $( \pm)$ & Less than 5 dots of $\alpha$-NB esterase \\
\hline Negative (-) & No $\alpha$-NB esterase dots in leukemic cells \\
\hline Modified $\alpha$-NB ac & \\
\hline Positive $(++)$ & More than 6 dots of $\alpha$-NB esterase in leukemic cells \\
\hline Weakly Positive (+) & Three to $5 \alpha$-NB esterase dots in leukemic cells \\
\hline Faint $( \pm)$ & One or $2 \alpha$-NB esterase dots in leukemic cells \\
\hline Negative (-) & No $\alpha$-NB esterase dots in leukemic cells \\
\hline
\end{tabular}

min, at $4^{\circ} \mathrm{C}$. After washing twice with PBS cells were subjected for flow cytometric analysis.

\section{Results}

\section{Patient characteristics, cytochem- istry, and tentative diagnosis of AML subtype}

In Table 2, clinical data and cytochemistry of 19 AML patients included in this study are shown. MPO staining was negative in 4 of the 19 patients examined. As for the $\alpha$-NB staining, 5, 8, 6, and 1 of the 19 patients showed positive, weakly positive, faintly positive, and negative staining, respectively. Patients in whom leukemic cells showed positive or weakly positive $\alpha$ NB staining could be diagnosed with AMLM5 because blast cells in individual patients showed morphologically monocytic differentiation. On the other hand, patients in whom leukemic cells showed faintly positive or negative $\alpha-\mathrm{NB}$ staining were tentatively diagnosed with AML-M5a or undifferentiated leukemia (Patient No.7, 9, 10, 13, and 14), indicating diagnostic difficulty with only morphological and cytochemical examinations for this group of patients. However, AML-M1, AML-M2, AML-M3, AML-M6,

Table 2. Clinical and laboratory data for the $19 \mathrm{AML}$ patients studied in the present study.

\begin{tabular}{|c|c|c|c|c|c|c|c|c|}
\hline No & Age & Sex & Specimen & Nec & $\%$ leukemic cells & Tentative diagnosis & POX & $\alpha-N B$ \\
\hline 1 & 79 & M & PB & 228.3 & 85.4 & AML-M5a & + & ++ \\
\hline 2 & 66 & M & $\mathrm{BM}$ & 45.4 & 35.8 & AML-M5a & + & + \\
\hline 3 & 66 & M & $\mathrm{BM}$ & 242.3 & 34.7 & AML-M4 & + & ++ \\
\hline 4 & 76 & $\mathrm{~F}$ & $\mathrm{BM}$ & 564.1 & 86.7 & AML-M5a & + & + \\
\hline 5 & 65 & M & BM & 98.9 & 63.7 & AML-M5a & + & + \\
\hline 6 & 42 & $\mathrm{~F}$ & $\mathrm{~PB}$ & 2.9 & 9.7 & AML-M5a & - & + \\
\hline 7 & 50 & $\mathrm{~F}$ & $\mathrm{BM}$ & 107.1 & 92.0 & AML-M5a or undifferentiated AML & - & \pm \\
\hline 8 & 89 & M & $\mathrm{BM}$ & 53.6 & 23.4 & AML-M5a & + & + \\
\hline 9 & 70 & $\mathrm{~F}$ & $\mathrm{BM}$ & 114.8 & 55.2 & AML-M5a or undifferentiated AML & - & - \\
\hline 10 & 25 & M & $\mathrm{BM}$ & $1,080.4$ & 85.0 & AML-M5a or undifferentiated AML & + & \pm \\
\hline 11 & 47 & $\mathrm{~F}$ & $\mathrm{BM}$ & 635.8 & 83.4 & AML-M5a & + & ++ \\
\hline 12 & 74 & M & $\mathrm{BM}$ & 130.8 & 30.8 & AML-M5a & + & \pm \\
\hline 13 & 71 & M & $\mathrm{BM}$ & 388.0 & 40.5 & AML-M5a or undifferentiated AML & - & + \\
\hline 14 & 60 & M & $\mathrm{BM}$ & 272.3 & 87.2 & AML-M5a or undifferentiated AML & + & \pm \\
\hline 14 & 60 & M & PB & 21.0 & 87.7 & AML-M5a & + & + \\
\hline 15 & 66 & M & $\mathrm{BM}$ & 343.7 & 62.1 & AML-M5a & + & + \\
\hline 16 & 94 & M & PB & 71.7 & 53.9 & AML-M5b & + & ++ \\
\hline 17 & 76 & $\mathrm{~F}$ & $\mathrm{BM}$ & 25.1 & 47.6 & AML-M5a & + & \pm \\
\hline 18 & 87 & $\mathrm{~F}$ & PB & 244.0 & 77.7 & AML-M5b & + & ++ \\
\hline 19 & 65 & M & $\mathrm{BM}$ & 13.7 & 34.2 & AML-M5a & + & \pm \\
\hline
\end{tabular}

No.: Patient number, POX: peroxidase staining, PB: peripheral blood, BM: bone marrow, Ncc: nucleated cell count and is expressed as $\times 10^{9} / \mathrm{L} .++$ : positive, + : weakly positive, \pm : faintly positive 
and AML-M7 could be ruled out from their characteristic morphology or negative naphthol AS-D chloroacetate esterase staining (for AML-M1, AML-M2, and AML-M3) in these 5 patients. In addition, positive $\alpha$-NB staining was diminished by the treatment with sodium fluoride $(\mathrm{NaF})$ in all cases.

\section{Phenotypes of the leukemic cells}

Surface antigens and cytoplasmic MPO (cyMPO) expressed in leukemic cells from the 19 AML patients are shown in Table 3. In all patients, leukemic cells showed the AML phenotype, that is, the concomitant expression of CD34, CD33, and CD13, although these cells lacked CD34 expression in Patients 1, 16, 17, and 18. HLA-DR was positive in all but Patient 7 (data not
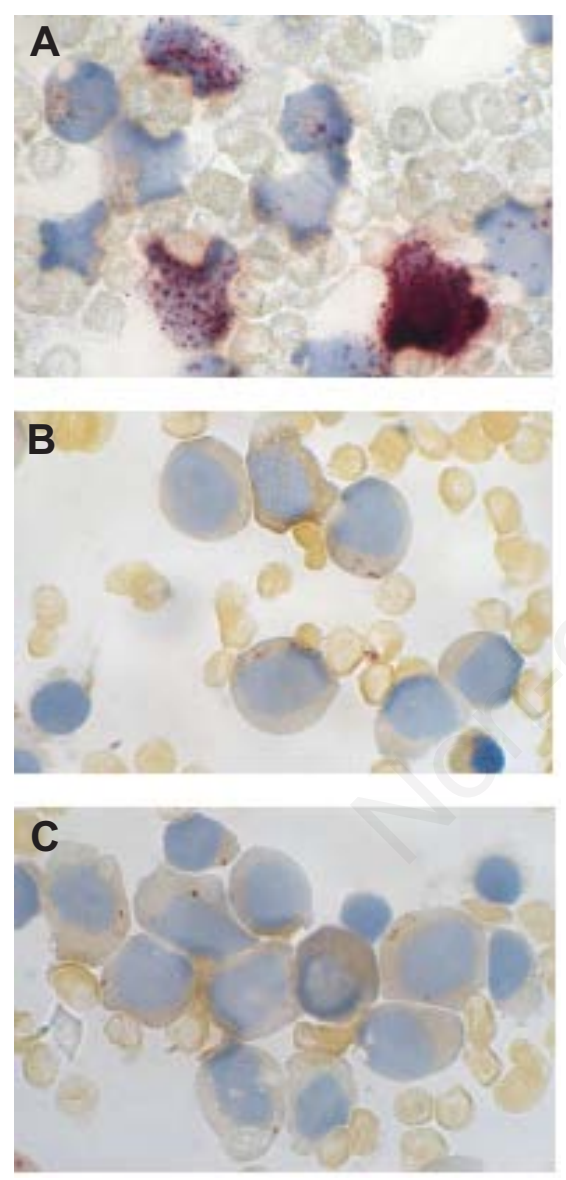

Figure 1. Alfa-naphthyl butyrate ( $\alpha-\mathrm{NB})$ esterase staining for bone marrow cells from patients with AML-M5 and criteria for $\alpha$-NB esterase activity by the present authors. A) positive $\alpha-\mathrm{NB}$ staining (H): a dot number of more than 6 in leukemic cells. B) Weakly positive $\alpha$-NB staining (+): 3 to 5 dots on leukemic cells. C) Faintly positive $\alpha-\mathrm{NB}$ staining $( \pm)$ : 1 to 2 dots on leukemic cells. shown). CyMPO was not detected in 4 patients (Patients 6, 7, 13, and 17). To rule out acute lymphoblastic leukemia (ALL), we performed CD19 staining in all but 2 patients (Patients 1 and 6) with positive staining (30.6\%) in Patient 10. In Patient 1, MPO staining was positive, and in Patient 6 , leukemic cells were weakly positive for $\alpha$-NB staining with monocytic morphology (Table 2). In Patient 10 whose leukemic cells exhibited CD19 positivity, these cells were positive for MPO staining (Table 2). In Patients 7, 13, 17, whose leukemic cells showed cyMPO negativity, CD19 was negative in all these patients. Therefore, ALL could be ruled out in all 19 patients included in the present study. Regarding the monocyte-related antigens, CD4 expression was observed in 9 of the 19 patients examined, CD11c in 16 of the 19 patients, and CD64 in 9 of the 16 patients. CD14 expression was observed in only 9 of all 19 patients. These results indicated that $\mathrm{CD} 11 \mathrm{c}$ was the most frequently expressed $(84.2 \%)$, followed by CD64 (56.3\%), CD4 (47.4\%), and CD14 (31.6\%). A representative flow cytometry of bone marrow cells from Patient 10 is shown in Figure 2, in which leukemic cells expressed both CD11c and CD64.

\section{Expression of monocyte-related antigens in AML-M1, -M2, and -M3}

We analyzed whether CD4, CD11c, CD14, or CD64 was expressed in AML in the neutrophil lineage. Leukemic cells were analyzed with the same antibody panel dur-
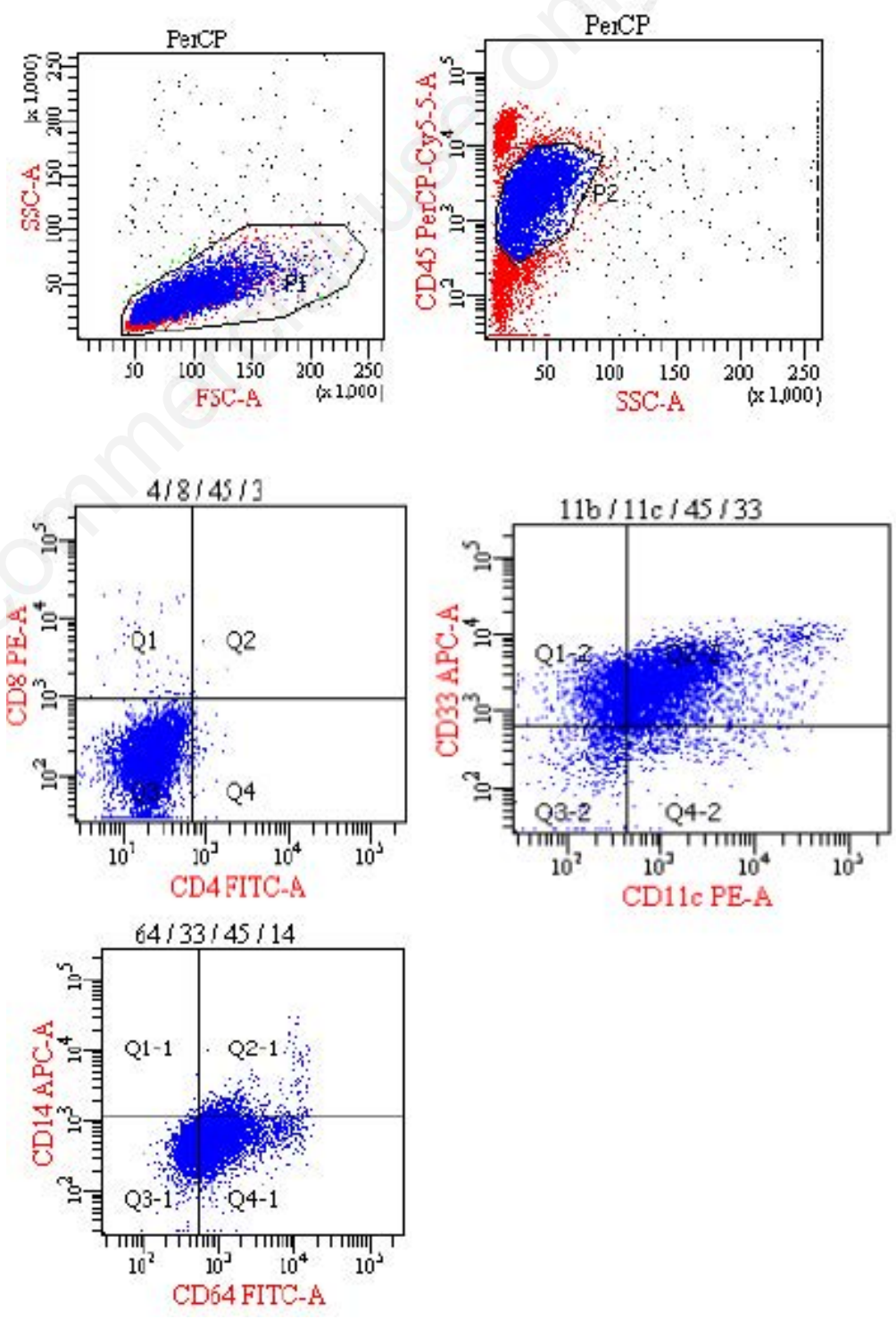

Figure 2. Flow cytometric diagrams from Patient 15 showing $15.5 \%$ CD11c- and $20.3 \%$ CD64-positive leukemic cells in CD45-gated mononuclear cells. 
ing the same period as in the present study in a total of 11 AML patients (2 AML-M1, 5 AML-M2, and 4 AML-M3 patients). Leukemic cells were positive for naphthol AS-D chloroacetate esterase staining in all these patients. CD11c was expressed in an AML-M1 patient at a level of $11 \%$, and CD64 was expressed in 2 AML-M2 patients (28 and 30\%) and one AML-M3 patient $(30 \%)$.

\section{Relationship between the expression of monocyte-related antigens and positive or weakly positive $\alpha-\mathrm{NB}$ staining}

In Table 4, the relationship between the expression of monocyte-related antigens and positive or weakly positive $\alpha$-NB staining is shown. Positive $\alpha$-NB staining was observed in 5 of the 19 patients (Patients 1, 3, 11, 16, and 18). In these patients, leukemic cells from 3 patients expressed all 4, Patient 2 expressed 2, and Patient 1 expressed one of the 4 monocyte-related antigens, respectively. Weakly positive $\alpha$ NB staining was observed in 8 of the 19 patients, and in all these 8 patients, the leukemic cells expressed one to 4 monocyte-related antigens. Collectively, leukemic cells that showed positive or weakly positive $\alpha$-NB staining mostly expressed more than 2 monocyte-related antigens, being consistent with the results of FCM and $\alpha$-NB staining.

\section{Relationship between the expression of monocyte-related antigens and faintly positive or negative $\alpha$-NB staining}

In Table 5, the relationship between the expression of monocyte-related antigens and faintly positive or negative $\alpha$-NB staining is shown. Faint $\alpha$-NB staining was observed in 6 of the 19 patients, and in all but one of these 5 patients (Patient 7), leukemic cells expressed one or 2 monocyte-related antigens, indicating a monocytic nature of the leukemic cells in these 5 patients. In Patient 7, leukemic cells expressed negative cyMPO staining (1.4\%) and showed both negative POX and naphthol AS-D chloroacetate esterase staining. Therefore, Patient 7 could be diagnosed with AML-M0. Negative $\alpha$-NB staining was observed in one patient (Patient 9). Patient 9 could be diagnosed with AML-M0 because of negative staining of monocyterelated antigens.

Collectively, the relationship between the AML subtype diagnosed by FCM and $\alpha$ NB activity of individual patients is shown for all 19 patients analyzed in the present study in Table 6 .

\section{Discussion}

To make a differential diagnosis of the AML subtype, double esterase staining is a useful tool. Naphthol AS-D chloroacetate esterase staining is specific for the granulocyte lineage and its sensitivity is excellent. ${ }^{1,3}$ While $\alpha$-NB staining is specific for the monocytic lineage, its sensitivity is too low for leukemic monocytes. ${ }^{1,3}$ Therefore, $\alpha$-NB staining often shows weakly or faintly positive results, even when leukemic cells are strongly suspected to be of a monocytic nature upon morphological examination. Indeed, in this study, positive or weakly positive $\alpha$-NB staining, which is usually accepted as diagnostic for AML-M5, was observed in only 13 of the 19 patients. We first confirmed whether positive or weakly positive $\alpha$-NB is sufficient for the diagnosis of AML-M5 with the expression of monocyte-related antigens with the use of FCM. As a result, the leukemic cells from these 13 patients expressed more than 2 monocyterelated antigens, except for 2 patients (Patients 7 and 14; CD11c only), indicating that positive or weakly positive $\alpha$-NB staining is sufficient to make a diagnosis of AML-M5.

The problem to be addressed in this study was the diagnostic value of faintly

Table 3. Expression of surface membrane antigens and cytoplasmic MPO in leukemic cells from 19 AML patients.

\begin{tabular}{lcccccccccccc} 
No. & CD13 & CD33 & CD34 & CD4 & CD7 & CD11c & CD11b & CD64 & CD14 & CD56 & M1P0 \\
1 & 84.9 & 98.9 & 4.0 & 0.1 & 91.7 & 93.1 & 27.6 & 2.2 & 2.0 & 2.0 & 34.9 \\
2 & 93.4 & 92.9 & 91.2 & 85.9 & 5.6 & 57.1 & 55.9 & 54.7 & 25.4 & 4.5 & 29.2 \\
\hline 3 & 55.7 & 99.5 & 19.8 & 2.3 & 7.5 & 92.4 & 73.8 & ND & 11.2 & 4.9 & 32.7 \\
4 & 95.1 & 98.9 & 86.3 & 0.1 & 87.6 & 84.8 & 34.8 & ND & 9.2 & 0.5 & 10.2 \\
\hline 5 & 82.4 & 98.0 & 89.1 & 12.3 & 1.1 & 70.7 & 23.6 & ND & 7.3 & 96.3 & 19.5 \\
6 & 23.1 & 19.0 & 61.8 & 59.5 & 7.0 & 49.6 & 14.1 & 3.2 & 0.9 & 0.7 & 3.7 \\
\hline 7 & 73.8 & 98.0 & 98.7 & 1.7 & 99.0 & 1.5 & 34.5 & 1.1 & 0.1 & 98.2 \\
8 & 95.3 & 54.0 & 85.2 & 8.4 & 61.3 & 25.1 & 28.6 & 25.8 & 9.8 & 3.9 \\
\hline 9 & 95.9 & 97.2 & 95.7 & 1.7 & 83.6 & 6.9 & 16.3 & 8.5 & 3.1 & 10.7 \\
10 & 68.7 & 79.8 & 77.2 & 0.8 & 80.4 & 51.3 & 95.8 & 75.6 & 3.8 & 68.2 & 47.0 \\
\hline 11 & 99.1 & 88.6 & 54.1 & 15.3 & 51.1 & 55.9 & 51.5 & 65.6 & 38.3 & 1.1 & 15.9 \\
12 & 86.0 & 96.5 & 95.9 & 0.5 & 91.8 & 35.6 & 12.6 & 16.9 & 1.4 & 3.4 & 75.1 \\
\hline 13 & 53.8 & 37.1 & 72.5 & 65.8 & 15.5 & 22.4 & 22.3 & 26.9 & 17.1 & 1.1 & 3.2 \\
$14(\mathrm{BM})$ & 11.5 & 69.9 & 98.5 & 2.5 & 1.0 & 30.4 & 10.8 & 3.2 & 0.7 & 0.2 & 94.4 \\
\hline $14(\mathrm{~PB})$ & 37.3 & 50.7 & 99.7 & 1.5 & 0.1 & 47.5 & 18.5 & 4.1 & 0.1 & 0.0 & 97.4 \\
15 & 78.3 & 93.8 & 82.6 & 2.0 & 74.3 & 15.7 & 19.4 & 16.8 & 5.2 & 0.9 & 74.6 \\
\hline 16 & 76.8 & 99.8 & 0.0 & 84.2 & 0.0 & 99.8 & 99.4 & 97.4 & 53.7 & 54.6 & 28.2 \\
17 & 93.7 & 95.1 & 6.0 & 50.4 & 1.6 & 3.6 & 18.2 & 9.3 & 3.5 & 7.5 & 6.8 \\
\hline 18 (PB) & 1.6 & 99.5 & 2.3 & 91.4 & 4.1 & 82.5 & 96.1 & 93.5 & 74.3 & 8.6 & 94.8 \\
19 & 82.8 & 85.0 & 87.6 & 43.6 & 35.3 & 6.4 & 10.8 & 6.6 & 0.5 & 28.5 & 25.9 \\
\hline
\end{tabular}

No.: Patient number, cyMPO: cytoplasmic myeloperoxidase. The values indicate percentage of individual antigen-expressing cells in CD45-gated mononuclear cells. ND: Not done. 
positive $\alpha$-NB staining for AML-M5. Faintly positive $\alpha-\mathrm{NB}$ staining was observed in 6 of the 19 patients in this study, and FCM analysis showed that leukemic cells from 5 of these 6 patients expressed one to 2 monocyte-related antigens, indicating that the majority of patients in whom leukemic cells showed faintly positive $\alpha$-NB staining could be diagnosed with AML-M5. Aberrant expression of monocyte-related antigen in these patients may be unlikely because other subtype AML except for AML-M5 and AML-M0 could be ruled out. In the present study, negative $\alpha$ NB staining was observed in only one patient. An early study described the presence of $\alpha$-NB staining-negative AML-5 or AML-M4; however, the diagnosis of these types of AML was performed based on morphologic and cytochemical methods at this time period. ${ }^{17}$ Thus, the relationship between the antigen profile of the leukemic cells and negative $\alpha$-NB staining should be examined in a larger number of patients in the future.

We employed CD4, ${ }^{4,10} \mathrm{CD} 11 \mathrm{c},{ }^{10,12,16}$ CD14, ${ }^{5-11,13-16}$ and CD645-15 antigens as monocyte-related antigens, and diagnosed a patient with AML-M5 when the leukemic cells expressed at least one of these antigens at the level of $10 \%$ in the present study. Of these antigens, CD4, CD14, and CD64 may have been established as monocyte-related antigens. ${ }^{4-16} \mathrm{CD} 11 \mathrm{c}$ is expressed on immature monocytes ${ }^{10,12,16}$ like CD4. CD11c is also expressed on granulocytes, but mainly on mature granulocytes. ${ }^{18}$ Therefore, it may reasonable to conclude that leukemic cells are an immature monocytic lineage based on the expression of CD11c. Furthermore the in the present study, AML in the granulocytic lineage was excluded by the use of naphthol AS-D chloroacetate esterase staining; therefore, CD11c may have been a valuable tool to determine if the leukemic cells were of monocytic lineage. Indeed, CD11c was weakly positive in only one patient among 11 patients with AML in the neutrophil-lineage (AML-M1-3), as described above.

\section{Conclusions}

In conclusion, AML-M5 diagnosed by FCM included all cases of AML with positive or weakly positive $\alpha$-NB staining and even the majority of those with faint $\alpha-\mathrm{NB}$ activity. Therefore, faintly positive $\alpha-\mathrm{NB}$ staining should not be regarded as negative staining. Negative $\alpha$-NB staining may rule out AML-M5, but further studies are required.
Table 4. Relationship between expression of monocyte-related antigens and positive or weakly positive $\alpha-\mathrm{NB}$ staining.

\begin{tabular}{lcccccc} 
No. & CD4 & CD11c & CD64 & CD14 & MPO & a-NB \\
1 & 0.1 & 93.1 & 2.2 & 2.0 & 34.9 & ++ \\
2 & 85.9 & 57.1 & 54.7 & 25.4 & 29.2 & + \\
\hline 3 & 2.3 & 92.4 & ND & 11.2 & 32.7 & ++ \\
4 & 0.1 & 84.8 & ND & 9.2 & 10.2 & + \\
\hline 5 & 12.3 & 70.7 & ND & 7.3 & 19.5 & + \\
6 & 59.5 & 49.6 & 3.2 & 0.9 & 3.7 & + \\
\hline 8 & 8.4 & 25.1 & 25.8 & 9.8 & 77.0 & + \\
11 & 15.3 & 55.9 & 65.6 & 38.3 & 15.9 & ++ \\
\hline 13 & 65.8 & 22.4 & 26.9 & 17.1 & 3.2 & + \\
$14(\mathrm{~PB})$ & 1.5 & 47.5 & 4.1 & 0.1 & 97.4 & + \\
\hline 15 & 2.0 & 15.7 & 16.8 & 5.2 & 74.6 & + \\
16 & 84.2 & 99.8 & 97.4 & 53.7 & 28.2 & ++ \\
\hline $18(\mathrm{~PB})$ & 91.4 & 82.5 & 93.5 & 74.3 & 94.8 & ++ \\
\hline
\end{tabular}

No.: Patient number, The values indicate percentage of individual antigen-expressing cells in CD45-gated mononuclear cells. ++: positive, + : weakly positive, \pm : faintly positive. ND: Not done

Table 5. Relationship between expression of monocyte-related antigens and faintly positive or negative $\alpha-\mathrm{NB}$ staining.

\begin{tabular}{lcccccc} 
No. & CD4 & CD11c & CD64 & CD14 & MPO & a-NB \\
7 & 1.7 & 1.5 & 1.1 & 0.1 & 1.4 & \pm \\
9 & 1.7 & 6.9 & 8.5 & 3.1 & 18.7 & - \\
\hline 10 & 0.8 & 51.3 & 75.6 & 3.8 & 47.4 & \pm \\
12 & 0.5 & 35.6 & 16.9 & 1.4 & 75.1 & \pm \\
\hline $14(\mathrm{BM})$ & 2.5 & 30.4 & 3.2 & 0.7 & 94.4 & \pm \\
17 & 50.4 & 3.6 & 9.3 & 3.5 & 6.8 & \pm \\
\hline 19 & 43.6 & 6.4 & 6.6 & 0.5 & 25.9 & \pm \\
\hline
\end{tabular}

The values indicate percentage of individual antigen-expressing cells in CD45-gated mononuclear cells. ++ positive, + : weakly positive, \pm : faintly positive. ND: Not done.

Table 6. Final AML subtype determined by the combination of monocyte-related antigen expression and $\alpha$-NB staining in the 19 AML patients studied in the present study.

\begin{tabular}{lcccccc} 
No. & Final AML subtype & CD4 & CD11c & CD64 & CD14 & a-NB \\
1 & AML-M5a & - & + & - & - & ++ \\
2 & AML-M5a & + & + & + & + & + \\
\hline 3 & AML-M4 & - & + & ND & + & ++ \\
4 & AML-M5a & - & + & ND & - & + \\
\hline 5 & AML-M5a & + & + & ND & - & + \\
6 & AML-M5a & + & + & - & - & + \\
\hline 7 & AML-M0 & - & - & - & - & \pm \\
8 & AML-M5a & - & + & + & - & + \\
\hline 9 & AML-M0 & - & - & - & - & - \\
10 & AML-M5a & - & + & + & - & \pm \\
\hline 11 & AML-M5a & + & + & + & + & ++ \\
12 & AML-M5a & - & + & + & - & \pm \\
\hline 13 & AML-M5a & + & + & + & + & + \\
$14(\mathrm{BM})$ & AML-M5a & - & + & - & - & \pm \\
\hline $14(\mathrm{~PB})$ & AML-M5a & - & + & - & - & + \\
15 & AML-M5a & - & + & + & - & + \\
\hline 16 & AML-M5b & + & + & + & + & ++ \\
17 & AML-M5a & + & - & - & - & \pm \\
\hline $18(\mathrm{~PB})$ & AML-M5b & + & + & + & + & ++ \\
19 & AML-M5a & + & - & - & - & \pm \\
\hline
\end{tabular}

No.: Patient number; + in each antigen indicates more than $10 \%$ of the cells expressed the individual antigens in CD45-gated mononuclear cells. ++ : positive, + : weakly positive, \pm : faintly positive. ND: Not done. 
CD45 gating in the subclassification of acute myeloid leukemias with monocytic differentiation. Am J Clin Pathol 1998;110:797-805.

1. Li CY, Lam KW, Yam LT. Esterases in human leukocytes. J Histochem Cytochem 1973;21:1-12.

2. Bozdech MJ, Bainton DF. Identification of alpha-naphthyl butyrate esterase as a plasma membrane ectoenzyme of monocytes and as a discrete intracellular membrane-bounded organelle in lymphocytes. J Exp Med 1981;153:18295.

3. Gamal AH. Classification of Acute Leukemia. INTECH. 2011. DOI: 10.5772/19848.

4. Zheng J, Wang X, Hu Y, et al. A correlation study of immunophenotypic, cytogenetic, and clinical features of 180 AML patients in China. Cytometry B Clin Cytom 2008;74:25-9.

5. Kaleem Z, Crawford E, Pathan MH, et al. Flow cytometric analysis of acute leukemias. Diagnostic utility and critical analysis of data. Arch Pathol Lab Med 2003;127:42-8.

6. Peters JM, Ansari MQ. Multiparameter flow cytometry in the diagnosis and management of acute leukemia. Arch Pathol Lab Med 2011;135:44-54.

7. Krasinskas AM, Wasik MA, Kamoun $\mathrm{M}$, et al. The usefulness of CD64, other monocyte-associated antigens, and to diagnostic hematopathology. Arch Pathol Lab Med 2004; 128:1004-22.

14. Dunphy $\mathrm{CH}$, Orton SO, Mantell J. Relative contributions of enzyme cytochemistry and flow cytometric immunophenotyping to the evaluation of acute myeloid leukemias with a monocytic component and of flow cytometric immunophenotyping to the evaluation of absolute monocytoses. Am J Clin Pathol 2004;122:865-74.

15. Yang DT, Greenwood JH, Hartung L, et al. Flow cytometric analysis of different CD14 epitopes can help identify immature monocytic populations. Am J Clin Pathol 2005;124:930-6.

16. Scott CS, Richards SJ, Master PS, et al. Flowcytometric analysis of membrane CD11b, CD11c and CD14 expression in acute myeloid leukaemia: relationships with monocytic subtypes and the concept of relative antigen expression. Eur J Haematol 1990;44:24-9.

17. Tomonaga M. Evaluation of FAB classification from cytomorphological and cytochemical view points. Rinsho Ketsueki 1982;23:1003-8.

18. Gorczyca W, Sun ZY, Cronin W, et al. Immunophenotypic pattern of myeloid populations by flow cytometry analysis. Methods Cell Biol 2011;103:221-66. 